\title{
Microquasars as sources of positron annihilation radiation
}

\author{
N. Guessoum ${ }^{1}$, P. Jean ${ }^{2}$, and N. Prantzos ${ }^{3}$ \\ 1 American University of Sharjah, College of Arts \& Sciences, Physics Department, PO Box 26666, Sharjah, UAE \\ e-mail: nguessoum@aus . edu \\ 2 CESR, CNRS/UPS, BP 4346, 31028 Toulouse Cedex 4, France \\ e-mail: Pierre.jean@cesr.fr \\ 3 Institut d'Astrophysique de Paris, 98bis Bd. Arago, 75104 Paris, France \\ e-mail: prantzos@iap.fr \\ Received 21 March 2006 / Accepted 28 June 2006
}

ABSTRACT

\begin{abstract}
We consider the production of positrons in microquasars, i.e. X-ray binary systems that exhibit jets frequently, but not continuously. We estimate the production rate of positrons in microquasars, both by simple energy considerations and in the framework of various proposed models. We then evaluate the collective emissivity of the annihilation radiation produced by Galactic microquasars and we find that it might constitute a substantial contribution to the annihilation flux measured by INTEGRAL/SPI. We also discuss the possible spatial distribution of Galactic microquasars, on the basis of the (scarce) available data and the resulting morphology of the flux received on Earth. Finally, we consider nearby "misaligned" microquasars, with jets occasionally hitting the atmosphere of the companion star; these would represent interesting point sources, for which we determine the annihilation flux and the corresponding light curve, as well as the line's spectral profile. We discuss the possibility of detection of such point sources by future instruments.
\end{abstract}

Key words. gamma rays: theory - line: formation - ISM: general - X-rays: binaries

\section{Introduction: galactic positron sources}

Ever since the first detection of the gamma-ray signature (511 keV radiation) of the annihilating positrons in the Galaxy in 1970 (Johnson et al. 1972), the problem of the birth, propagation, interaction, and annihilation of the positrons has been a major topic of astrophysical investigation, both observationally and theoretically. Nonetheless, some of its fundamental issues, particularly the questions of positron origin and propagation, have remained mostly unsolved puzzles.

A series of observational campaigns, conducted by balloons ${ }^{1}$ in the first two decades and then by satellites ${ }^{2}$, have produced increasingly more precise, high-resolution spectra. Recent data have allowed a determination of the physical conditions in the interstellar medium where the positrons annihilate (Guessoum et al. 2004; Churazov et al. 2005; Jean et al. 2006), based on an improved understanding of the various physical processes involved (Guessoum et al. 2005). In parallel, considerable progress has been made in mapping the Galaxy at $511 \mathrm{keV}$, first by CGRO-OSSE (Purcell et al. 1994; Cheng et al. 1997; Purcell et al. 1997; Milne et al. 2000; Milne et al. 2001) and, in particular, recently by INTEGRAL-SPI (Knödlseder et al. 2005).

Despite that substantial progress, the origin of the huge amounts of positrons produced in the Galaxy $\left(10^{43} \mathrm{e}^{+} \mathrm{s}^{-1}\right)$ is still eluding us. A large number of potential sources has been proposed over the years: cosmic ray interactions with the interstellar medium (Ramaty et al. 1970), pulsars (Sturrock 1971), radioactive nuclei produced by explosive nucleosynthesis in supernovae (Clayton 1973) or novae (Clayton \& Hoyle 1974), compact objects housing either neutron stars or black holes (Ramaty \& Lingenfelter 1979), matter expelled by red giants

1 The pioneering Rice University group's campaign, the Bell-Sandia group's, GRIS, HEXAGONE.

${ }^{2}$ HEAO 3, CGRO-OSSE, WIND-TGRS, INTEGRAL-SPI.
(Norgaard 1980) and Wolf-Rayet stars (Dearborn \& Blake 1985; Prantzos \& Cassé 1986), gamma-ray bursts (Lingenfelter \& Hueter 1984), (light) dark matter (Rudaz \& Stecker 1988; Boehm et al. 2004), low-mass X-ray binaries (Prantzos 2004), hypernovae (Cassé et al. 2004), millisecond pulsars (Wang et al. 2006), and, most recently, pair production from the collision of gamma-ray photons from "SMall Mass Black Holes" (SMMBHs) and X-ray photons from the Galactic Center black hole Sgr A* (Titarchuk \& Chardonnet 2006).

The recent mapping of the Galaxy at $511 \mathrm{keV}$ (Knödlseder et al. 2005), particularly the very large Bulge/Disc (B/D) ratio inferred, has placed rather severe constraints on the positron candidate sources. Of the aforementioned potential sources, Type Ia supernovae, low-mass X-ray binaries (LMXB's), and dark matter have emerged, for various reasons, as possible (or, rather, not impossible) candidates. However, each of these has its own difficulties:

1) although the total positron emissivity of SNIa in the Galaxy matches the observed one, the corresponding bulge emissivity is estimated (e.g. Prantzos 2004; Diehl et al. 2005) to be an order of magnitude lower than required by SPI observations. Even assuming that large systematic uncertainties affect that estimate, the expected galactic distribution of SNIa does not correspond to the SPI B/D ratio, unless additional assumptions are made (e.g. important number of currently undetected SNIa in the galactic bulge, or transfer of a large fraction of the disk positrons to the bulge through the galactic magnetic field, see Prantzos 2006);

2) low-mass X-ray binaries (LMXBs) display a spatial distribution considerably peaked toward the Galactic centre region (Grimm et al. 2002), and their global energetics allow for a substantial positron production galaxywide (Prantzos 2004). However, the brightest of them (in X-rays) lie in the disc, not in the bulge of the Milky Way 
(Prantzos 2004); if positron emissivity correlates with X-ray emissivity (as is commonly assumed), LMXB's should be excluded on the basis of inadequate morphology;

3) dark matter particles as sources of Galactic positrons may also suffer from "morphology" problems, or at least uncertainties (see Ascasibar et al. 2005).

Finally, we must note the important constraint that Beacom \& Yüksel (2006) have pointed out w.r.t. all models in principle: if the positrons are relativistic $(E \gtrsim 3 \mathrm{MeV})$, then the annihilation in flight of a fraction of them (which is very large when the medium is neutral) will produce continuum photons of high energies ( $>$ a few $\mathrm{MeV}$ ) that could be detected by instruments such INTEGRAL and COMPTEL. This result places strong constraints on models, particularly those that have positrons produced at high energies and annihilating "in flight", i.e. while slowing down.

In the case of LMXBs, positrons should be produced as $\mathrm{e}^{+}-\mathrm{e}^{-}$pairs in the inner regions of their accretion discs. Some of these would annihilate locally, but a non-negligible fraction would be channeled out by jets - when these exist. In general, positrons would reach the ISM relatively far from their source, and they would propagate and annihilate, contributing to the diffuse $511 \mathrm{keV}$ emission. In special cases, when the jets are strongly inclined ("misaligned") toward the plane of the binary system, positrons could periodically hit the atmosphere of the companion star where they would produce an annihilation signature, characterized, in particular, by its time variability and line profile.

In this paper we consider the possible fate of positrons ejected by microquasar jets. We first estimate the rate of positron production in jets, by reviewing estimates from other studies and by using simple energetics considerations; in that respect, we pay special attention to upper limits derived from the INTEGRAL-SPI measurements of positron annihilation fluxes from point sources in the Galaxy. We then consider two possible consequences: 1) the collective microquasar contribution to the flux of galactic annihilation radiation, which we find to be potentially substantial (especially from the central regions); and 2) the annihilation line signature of "misaligned" microquasars. In the latter case, we study the fate of positrons in the companion's atmosphere in order to compute the photon emissivity, and determine the characteristic "light curve" of such a source; we find that interesting observational signatures could be obtained from close enough and/or strongly active sources.

In Sect. 2 we present a brief overview of X-ray binaries, microquasars, and jets. In Sect. 3 we present our estimates of the rate of positrons produced by microquasar jets. In Sect. 4 we consider the overall contribution of microquasars to the global galactic annihilation flux. In Sect. 5 we consider the annihilation of positrons in misaligned microquasars. In Sect. 6 we summarize our conclusions.

\section{X-ray binaries, microquasars, and jets}

X-ray binaries (XRB's) are systems containing a compact object (either a neutron star or a stellar-mass black hole) accreting matter from a companion star. A total of 280 galactic X-ray binaries are currently known: 131 High Mass X-ray Binaries (HMXB's) (Liu et al. 2000), in which the companion star has a mass $\geq 5$ solar masses (spectral type $\mathrm{O}$ or $\mathrm{B}$ ) and where the mass transfer usually takes place by way of the strong stellar wind; and 149 Low Mass X-ray Binaries (LMXB's)
(Liu et al. 2001), in which the companion has a low mass (spectral type later than B), and the mass transfer is carried out by Roche lobe overflow. Grimm et al. (2002) have shown that HMXB's tend to be distributed along the galactic plane, while LMXB's tend to be clustered in low Galactic longitudes. The number of XRB's brighter than $2 \times 10^{34} \mathrm{erg} / \mathrm{s}$ is estimated to $\sim 700$ in the Galaxy (Grimm et al. 2002; Paredes 2005).

Among the detected XRB's, 43 have now been found to exhibit radio emission, which is interpreted as synchrotron radiation. It is generally believed that the radio emission is evidence of jets. Of the 43 objects, 35 are LMXB's and 8 are HMXB's. Moreover, of the 43 systems, 16 are confirmed cases with resolved jets (Ribó 2005; Paredes 2005; Chaty 2005). Such systems (a compact object accreting from a companion star and ejecting a stream of relativistic particles) are known as microquasars. It is likely that all 43 radio-emitting X-ray binaries (REXB) are microquasars. The population of microquasars in the Galaxy is estimated at about 100 (Paredes 2005), although only 16 are "officially" known (or confirmed) at present. We note that Pandey et al. (2006a,b) have recently reported 4 new REX sources: IGR J17091-3624, IGR J17303-0601, IGR J17464-3213, and IGR J18406-0539; in addition, two sources, X Nor X-1 and IGR J17418-1212, have been presented as microquasars (Klein-Wolt et al. 2004; and Tsarevsky 2004, respectively). We refer to these six sources as "new tentative microquasars". The catalog of microquasars is expected to grow rapidly with the array of instruments and studies now investigating these objects (from radio to gamma rays).

Although jets of such systems were first observed in 1979 (in the peculiar object SS 433), microquasars were identified and imaged in the Galaxy only in 1992 when Mirabel et al. (1992) performed high-resolution radio observations of the GC's "great annihilator" 1E 1740.7-2942 and soon afterwards in GRS 1915+105 (Mirabel \& Rodriguez 1994).

Recent studies (Falcke et al. 2004; Mirabel 2004; Fender et al. 2004, 2005a) considerably improved our understanding of the conditions that lead to the emergence of jets in XRBs. First and foremost, a connection has been established between X-ray flux and jet formation: jets seem to appear when the accretion disc X-ray luminosity is low. Jets are apparently produced when the inner disc is replenished; there is a clear general pattern of steady jets in the "low/hard state" of the X-ray (microquasar) sources, while no jet is seen in "the high/soft state".

A relatively elaborate model of this scenario has been proposed by Fender et al. (2004, 2005a). A relation between the power in the jet (when it exists) and the X-ray luminosity of a given source is derived: $L_{\text {jet }}=A_{\text {steady }} L_{\mathrm{X}}^{0.5}$, with luminosities expressed in units of the corresponding Eddington luminosity. These authors argue that steady jets are produced when the X-ray spectrum of a source hardens beyond a certain value (which may be universal or vary somewhat from one source to another). The spectrum softens when the X-ray luminosity increases above about $1 \%$ of the Eddington value; when this happens, the jet first increases in speed and then quickly gets suppressed and disappears.

There seems to be an agreement on the above relation between $L_{\mathrm{jet}}$ and $L_{\mathrm{X}}^{0.5}$, which is supposed to hold for sources individually. On the other hand, there seems to be considerable uncertainty over the value of $A_{\text {steady }}$ (Fender et al. 2005b); indeed, using various sources (e.g. XTE J1118+480) for "calibration", authors obtain values ranging from 0.006 as a lower limit (Fender et al. 2005a) to 0.3 (Malzac et al. 2004), which Fender et al. (2005a) take as an upper limit. In the case of transient jets, Fender et al. (2005b) conclude that the value of $A_{\text {trans }}$ would 
range between 0.04 and 4.0, at least for black hole sources, which tend to exhibit jets that are about 10 times more powerful than those of neutron star jets, both in the steady and in the transient cases.

Secondly, a general correlation is also found between the velocity of the outflow and the X-ray luminosity of the accreting source: increases in X-ray luminosity tend to accelerate the jets as long as the source remains in the low/hard state. Fender et al. (2005a) further argue that the velocities of transient jets are significantly larger $(\gtrsim 0.87 \mathrm{c})$ than those of steady jets $(\lesssim 0.7 \mathrm{c})$. Jets with larger Lorentz factors have also been considered, but the standard cases are those presented by Fender et al. (2005a).

The particle content of the jets is among the major unresolved issues in the field of microquasars (e.g. Scheck et al. 2002). Observations of emission lines in the case of SS 433 (Marshall et al. 2002) suggest a substantial baryonic content of its jets. Several studies have considered the implications of a hadronic jet composition, either for the galactic cosmic ray content (Heinz \& Sunyaev 2002; Fender et al. 2005b) or for the synthesis of $\mathrm{Li}$ on the surface of the companion star (Butt et al. 2003). Others have considered the gamma-ray and neutrino production at the surface of the companion by impinging highenergy protons (Romero \& Orellana 2005). On the other hand, arguments for $\mathrm{e}^{+}-\mathrm{e}^{-}$pair-dominated jets have been put forward, especially in cases involving the extraction of the spin energy of the black hole (Celotti \& Blandford 2001). A strong argument in favor of such a leptonic composition is the repeated observations of highly polarized jets. Several leptonic microquasar models have been proposed in recent times, e.g. Bosch-Ramon \& Parades (2004a,b), Bosch-Ramon et al. (2005), Dermer \& Böttcher (2006); see the review by Romero (2005). Some rather complex models have also been proposed, like "two-flow" models (pair beam surrounded by a mildly relativistic $\mathrm{e}^{-}-\mathrm{p}$ plasma) , or $\mathrm{e}^{-}-\mathrm{p}$ jets that later get loaded with pairs by interactions with high-energy photons (e.g. Scheck et al. 2002, and references therein).

In this work we consider microquasar jets channeling positrons (or $\mathrm{e}^{+}-\mathrm{e}^{-}$pairs) from the inner regions of the source's accretion disc into the ISM or towards the companion star (if the jet is "misaligned"). We also note that positrons can also be produced by hadrons from the jet colliding with nuclei (e.g. through $\mathrm{p}-\mathrm{p} \rightarrow \mathrm{p}+\mathrm{n}+\pi^{+}$), but such processes would contribute only negligible amounts of positrons due to the small numbers of high-energy hadrons as well as the low values of the relevant cross sections.

In order to estimate the flux of the resulting annihilation radiation, the rate of positron ejection by the jets must then be determined.

\section{Rate of positrons produced by microquasar jets}

The high-temperature, high-density conditions in the inner regions of the binary system's accretion disc produce electronpositron pairs via $\gamma+\gamma \longrightarrow \mathrm{e}^{+}+\mathrm{e}^{-}$reactions. A fraction of them annihilate close to the compact object, but when jets appear, they may channel out a significant number of pairs. A few authors have attempted to model and estimate the production and ejection of pairs in those conditions.

According to Beloborodov (1999) the pairs are cooled to energies of $1-10 \mathrm{keV}$ and blown away by soft radiation to form a semi-relativistic wind. Depending on the compactness of the source ( $l=L \sigma_{\mathrm{T}} / m_{\mathrm{e}} c^{3} R$, where $L$ and $R$ are the power and radius of the emitting region, and $\sigma_{\mathrm{T}}$ is the Thomson cross section), the plasma will form an optically thin or thick atmosphere. The density of the outflow and the rate of ejection of electrons and positrons depend on the rate of production of pairs (which, in turn, depend on the photon "seed" spectrum and on the accretion disc model), the annihilation rate and the "escape efficiency" of the pairs. Under the assumption of an optically thin pair wind, where the pairs escape before they annihilate $\left(\tau_{\text {esc }}<\tau_{\text {ann }}\right.$, with $\tau_{\text {ann }} \sim 1 / n_{\mathrm{e}} \sigma_{\mathrm{T}} c$, where $n_{\mathrm{e}}$ is the electron density), Beloborodov (1999) shows that the maximum pair luminosity is given by

$L_{\mathrm{e}^{+} \mathrm{e}^{-}}^{\max }=\frac{2 \pi m_{\mathrm{e}} c^{3} R}{\sigma_{\mathrm{T}}}$

which translates into a rate of pair injection in the jet of $\sim 4 \times$ $10^{41} \mathrm{~s}^{-1}$. A substantial fraction of these pairs, perhaps up to $90 \%$ (as argued by Misra \& Melia 1993), would annihilate near the base of the jet, producing a broad and redshifted line (that is rather difficult to detect); as many as $\sim 10^{41} \mathrm{~s}^{-1}$ positrons are then expelled into the ISM generally or, occasionally, in the direction of the companion star.

Misra \& Melia (1993) suggest that the intense radiation field is responsible for the Compton acceleration of the pairs produced in the inner regions of the accretion disc. They find that a large rate of pairs (up to $6 \times 10^{42} \mathrm{~s}^{-1}$ ) stream outwards from the disc (at velocities of $\sim 0.7 \mathrm{c}$ ), even after $90 \%$ have annihilated near the base. One must note, however, that this large rate is obtained with an accretion rate of $\sim 5 \times 10^{-8} M_{\odot} \mathrm{yr}^{-1}$, a rate that can normally be attained only in episodic outbursts. Indeed, the model of Misra \& Melia (1993) was mainly aiming to reproduce the 1E 1740.7-2942 "annihilation flare" of 1991.

Yamasaki et al. (1999) consider two-temperature accretion discs by taking into account the formation of relativistic pair outflows, in both AGN and microquasars. They show that in the inner regions of the discs, when the mass accretion rate becomes larger than about one tenth of the Eddington rate $\left(1.4 \times 10^{17} M / M_{\odot} \mathrm{g} \mathrm{s}^{-1}\right.$ where $M$ is the mass of the compact object) or $\sim 2 \times 10^{-10} M_{\odot} \mathrm{yr}^{-1}$, most of the viscously dissipated energy is converted into the thermal and kinetic energy of the electron-positron pairs. They obtain a maximum power of the pair outflow of $0.136 L_{\mathrm{Edd}}$ for an accretion rate of $10^{-9} M_{\odot} \mathrm{yr}^{-1}$ (assuming $M=1 M_{\odot}$ and $R=10 R_{\odot}$ ), which translates into a pair ejection rate of $2 \times 10^{42} \mathrm{~s}^{-1}$, assuming the jet is leptonic.

Considering the global energetics of microquasar jets in the Galaxy, one may note that

- at $L_{X}=0.5 L_{\text {Edd }}$ one obtains $\dot{N}_{\mathrm{e}^{+}}=2 \times 10^{42} \mathrm{~s}^{-1}$ (Yamasaki et al. 1999), which in the following estimate we may use as a "yardstick";

- steady jets are produced at $L_{X}=0.01-0.1 L_{\text {Edd }}$ (Fender et al. 2004, 2005a);

and

- $\dot{N}_{\mathrm{e}^{+}} \propto L_{\mathrm{jet}} \propto L_{\mathrm{X}}^{0.5}$, where the value of the proportionality constant $A$ is not needed if one uses the above "yardstick";

one can infer that the positron emissivity of a jet in the steady state lies in the range $\dot{N}_{\mathrm{e}^{+}} \sim 4 \times 10^{40}-9 \times 10^{41} \mathrm{e}^{+} \mathrm{s}^{-1}$, with $10^{41} \mathrm{e}^{+} \mathrm{s}^{-1}$ as a reasonable average value.

Finally, in attempting to fit the Nova Muscae 1991 "annihilation flare", Kaiser \& Hannikainen (2002) inferred a positron annihilation rate of $2 \times 10^{43} \mathrm{~s}^{-1}$ in the atmosphere of the companion star (assuming a distance to Earth of $\approx 5.5 \mathrm{kpc}$ ). They argued that such a high pair yield can be achieved when the plasma is "photon-starved", that is when the number of high-energy photons in the disc strongly exceeds that of the soft seed photons (see Zdziarski et al. 1990).

Despite the wide range in the results obtained in the aforementioned studies, one concludes that a reasonable estimate for 
Table 1. Limits on positron rates from SPI upper limits for XRB sources of interest. The positron rates were calculated assuming a positronium fraction of $95 \%$. The bottom part of the table lists microquasar sources, the last two or three being misaligned ones.

\begin{tabular}{|c|c|c|c|c|c|c|}
\hline Source & Type & $\begin{array}{l}\text { Distance } \\
(\mathrm{kpc})\end{array}$ & $\begin{array}{r}l \\
(\mathrm{deg})\end{array}$ & $\begin{array}{r}b \\
(\operatorname{deg})\end{array}$ & $\begin{array}{c}3 \sigma \text { flux limit } \\
\left(10^{-4} \mathrm{~cm}^{2} \mathrm{~s}^{-1}\right)\end{array}$ & $\begin{array}{l}\text { Positron rate } \\
\left(\mathrm{e}^{+} \mathrm{s}^{-1}\right)\end{array}$ \\
\hline GX $349+2$ & LMXB & $\lesssim 10$ & 349.1 & 2.75 & 0.8 & $\gtrsim 1.7 \times 10^{42}$ \\
\hline GX 5-1 & LMXB & 8 & 5.08 & -1.02 & 0.7 & $9.4 \times 10^{41}$ \\
\hline Nova Muscae & LMXB & 3 & 295.3 & -7.07 & 2 & $3.8 \times 10^{41}$ \\
\hline A $0620-00$ & LMXB & 2 & 209.96 & -6.54 & 3.8 & $3.1 \times 10^{41}$ \\
\hline Cen X-4 & LMXB & 1.2 & 332.24 & 23.89 & 1.7 & $5.2 \times 10^{40}$ \\
\hline GRS $1915+105$ & LMXB & 12.5 & 45.37 & -0.22 & 1 & $3.3 \times 10^{42}$ \\
\hline Cir X-1 & LMXB & 10 & 322.12 & 0.04 & 1.1 & $2.3 \times 10^{42}$ \\
\hline Cyg X-3 & HMXB & 9 & 79.85 & 0.7 & 1 & $1.7 \times 10^{42}$ \\
\hline 1E $1740.7-2942$ & LMXB & 8.5 & 359.1 & -0.11 & 0.9 & $1.4 \times 10^{42}$ \\
\hline GRS 1758-258 & LMXB & 8.5 & 4.51 & -1.36 & 0.7 & $1.1 \times 10^{42}$ \\
\hline GX 339 & LMXB & $\gtrsim 8$ & 0.68 & -0.22 & 0.8 & $\lesssim 10^{42}$ \\
\hline SS 433 & HMXB & 4.8 & 39.69 & -2.24 & 0.9 & $4.5 \times 10^{41}$ \\
\hline LS 5039 & HMXB & 2.9 & 16.88 & -1.29 & 0.9 & $1.5 \times 10^{41}$ \\
\hline Sco X-1 & LMXB & 2.8 & 359.1 & 23.78 & 1.5 & $2.4 \times 10^{41}$ \\
\hline Cyg X-1 & HMXB & 2.5 & 71.33 & 3.07 & 1 & $1.4 \times 10^{41}$ \\
\hline XTE J1118+480 & LMXB & 2.5 & 157.66 & 62.32 & 4.5 & $5.9 \times 10^{41}$ \\
\hline $\mathrm{LS} \mathrm{I}+61^{\circ} 303$ & HMXB & 2 & 135.68 & 1.09 & 3.3 & $2.8 \times 10^{41}$ \\
\hline IGR J17091-3624 & $?$ & $8.5 ?$ & -10.48 & 2.21 & 0.7 & $1.1 \times 10^{42}$ \\
\hline IGR J17303-0601 & LMXB & $8.5 ?$ & 17.93 & -1.61 & 0.9 & $1.4 \times 10^{42}$ \\
\hline IGR J17464-3213 & LMXB & $8.5 ?$ & -2.87 & 15.01 & 0.9 & $1.4 \times 10^{42}$ \\
\hline IGR J18406-0539 & $?$ & $8.5 ?$ & 26.67 & -0.17 & 1 & $1.5 \times 10^{42}$ \\
\hline X Nor X-1 & LMXB & $8.5 ?$ & -23.09 & 0.25 & 1 & $1.6 \times 10^{42}$ \\
\hline IGR J17418-1212 & $?$ & $8.5 ?$ & 13.93 & 9.41 & 0.9 & $1.4 \times 10^{42}$ \\
\hline XTE J1550-564 & Misaligned? & 5.3 & -34.12 & -1.83 & 1.1 & $6.6 \times 10^{41}$ \\
\hline V4641 Sgr & Misaligned & 9.6 & 6.77 & -4.79 & 0.7 & $1.4 \times 10^{42}$ \\
\hline GRO J1655-40 & Misaligned & 3.2 & -15.02 & 2.46 & 0.8 & $1.7 \times 10^{41}$ \\
\hline
\end{tabular}
"steady-state" production and ejection of pairs in normal condi-
tions is $\sim 10^{41} \mathrm{~s}^{-1}$.

Taking a different, empirical approach, interesting upperlimit constraints to the microquasar positron emissivity may be established by way of the recent annihilation radiation measurements by INTEGRAL-SPI. Knödleseder et al. (2005) have published 3- $\sigma$ flux upper limits for a dozen galactic microquasars/LMXB's/HMXB's; we have completed the data for other sources of interest to us here, particularly the "misaligned" microquasars that we will consider in some detail in Sect. 5. Table 1 lists the sources we have considered, with the corresponding SPI upper-limits on their $511 \mathrm{keV}$ emission flux (taking the sources to be point-like) and inferred rates of positron injection, assuming that positrons do not annihilate far from the source. The bottom part of the table lists microquasar sources, the last two or three being misaligned ones.

It can be seen that the data of the Table imply that upper limits for the steady positron annihilation rate of individual sources are always $>10^{41} \mathrm{e}^{+} \mathrm{s}^{-1}$ (albeit with large uncertainties, due in particular to uncertain distance estimates), so the upper-limit rates inferred from INTEGRAL data do not clash with the average value derived in the works cited above.

From the different considerations and estimates presented above, it appears that a "canonical average" rate of $\sim 10^{41} \mathrm{e}^{+} \mathrm{s}^{-1}$ ejected by the microquasar jets is not unreasonable. We will adopt this value for the determination of expected fluxes from individual sources (Sect. 5), but realizing the corresponding large uncertainty, we will also consider lower $\left(10^{40} \mathrm{e}^{+} \mathrm{s}^{-1}\right)$ values for "weak-episode jets" as well as larger $\left(10^{42} \mathrm{e}^{+} \mathrm{s}^{-1}\right)$ ones, in "strong flare" cases. We immediately note that should this average, "canonical value" prove to be reasonably correct, the $\sim 100$ microquasars that are believed to exist in the Galaxy (Paredes 2005) would produce a global annihilation emissivity near that measured by SPI (and previous instruments).

Another upper limit on the collective emission by galactic microquasars may be derived by considering the global energetics. Indeed, (i) the total luminosity of LMXRBs in the Milky Way is $2-3 \times 10^{39} \mathrm{erg} \mathrm{s}^{-1}$ (Grimm et al. 2002); while (ii) the luminosity of $\sim 10^{43} \mathrm{e}^{+} \mathrm{s}^{-1}$ observed by SPI/INTEGRAL is $\sim 10^{37} \mathrm{erg} \mathrm{s}^{-1}$, assuming the positronic jets are mildly relativistic (i.e. positrons have energies $<1 \mathrm{MeV}$ ). Of course, as Grimm et al. (2002) note, the Galactic LMXRB population is dominated by a dozen bright sources (of $\sim 10^{38} \mathrm{erg} \mathrm{s}^{-1}$ each) lying in the disk. But the remaining fraction of $\sim 90 \%$ is highly clustered towards the bulge (Fig. 1 in Grimm et al. 2002), as required by the SPI data and noted in Prantzos (2004), while their collective luminosity is $2-3 \times 10^{38} \mathrm{erg} \mathrm{s}^{-1}$, i.e. 20 times larger than required to explain the Galactic positron energetics. We note that those are precisely the low-luminosity (sub-Eddington) sources that may produce jets (see the discussion in Sect. 2). According to estimates by Paredes (2005), based on current microquasar statistics, there are about 100 microquasars in the Milky Way, which corresponds to about $1 / 3$ of the $\sim 300$ LMXRBs in our Galaxy as estimated by Grimm et al. (2002). Applying this correction factor of $1 / 3$ to the microquasar energetics, still leaves about $10^{38} \mathrm{erg} \mathrm{s}^{-1}$ available for positron production in their jets, i.e. about 6 times more than required by SPI data. We note at this point that some of the currently observed microquasars result from HMXRBs, but their fraction is rather small (less than $20 \%$ ) and does not affect the arguments presented here (see next section for a more detailed treatment). Moreover, we wish to stress the large (order-of-magnitude) uncertainty that exists in 
our current knowledge of the ratio between the energy that goes into positrons or into the jet (as a whole) compared to the energy that is radiated, an issue that is further complicated by the unknown lepton-to-hadron content ratio of the jet.

The simple estimate made in the previous paragraph implies that the ratio between the positron power and the X-ray luminosity of microquasars is, on average, about $16 \%$. One must recall, however, that there is a relation between the two quantities, namely $\dot{N}_{\mathrm{e}^{+}} \sim L_{\mathrm{jet}} \sim L_{\mathrm{X}}^{1 / 2}$ (references given above), which leads to more realistic expectations.

Indeed, taking the derivative of the cumulative X-ray luminosity function given by Grimm et al. (2002) for LMXRB's (see Eq. (15) of Grimm et al. 2002) and assuming that $1 / 3$ of LMXRBs are microquasars, one can derive a differential luminosity distribution function for microquasars:

$\mathrm{d} N / \mathrm{d} L_{\mathrm{X}}=2.6 L_{\mathrm{X}}^{-1.26}$,

where $L_{\mathrm{X}}$ is in Eddington units $\left(1.3 \times 10^{38} \mathrm{erg} / \mathrm{s}\right)$ and relates to $L_{\mathrm{e}^{+}}$as $L_{\mathrm{e}^{+}}=B L_{\mathrm{X}}^{a}$, with $a=1$ for a linear relationship and $a=1 / 2$ for the alternate, more realistic relation.

Now if the total positron power in the Galaxy is required to be $10^{37} \mathrm{erg} / \mathrm{s}$ (i.e. 0.1 in Eddington units), and using the above equation (Eq. (2)) to integrate for the total power, one finds that for $a=0.5, B$ is between 0.9 and $1.6 \%$ depending on the assumption made on the maximum X-ray luminosity of the microquasar ( 1 or $0.1 L_{\text {Edd }}$, respectively), which are very reasonable figures.

Furthermore, having estimated $B$ and thus obtained an analytic distribution function for the microquasars' positron emissivity, one can obtain the total rate of positrons emitted by all microquasars (of different luminosities) in the Galaxy by integrating $\mathrm{d} N / \mathrm{d} L_{\mathrm{X}}$ against the luminosities and the rate of positrons emitted by each source, as obtained by Yamasaki et al. (1999). Again, for the linear relation $(a=1)$ one obtains a total rate $N_{\mathrm{e}^{+}, \text {tot }}$ between 0.3 and $1.4 \times 10^{43} \mathrm{e}^{+} / \mathrm{s}$ (depending on the maximum X-ray luminosity of a given microquasar, i.e. 0.1 or $1 L_{\mathrm{Edd}}$, respectively); however, for the preferred non-linear relationship $(a=1 / 2)$, one obtains $N_{\mathrm{e}^{+}}$,tot between 1.8 and $3.1 \times 10^{43} \mathrm{e}^{+} / \mathrm{s}$ (microquasar X-ray luminosities of 0.1 and $1 L_{\text {Edd }}$, respectively), values that are very encouraging in considering microquasars as possible contributors to the overall positron annihilation flux from the central galactic regions.

\section{Contribution of microquasars to the galactic positron annhilation radiation}

Assuming that $\sim 100$ microquasars exist in the Galaxy (Paredes 2005), and noting from the locations plot of the 22 microquasars presently known/confirmed (Fig. 1) that about half of them are in the central regions, i.e. $\sim \pm 25$ degrees from the $\mathrm{GC}$, we can estimate the flux of annihilation radiation that can be expected from such a population of sources with jets ejecting $\approx 10^{41} \mathrm{e}^{+} \mathrm{s}^{-1}$ on average and compare it to the global galactic centre annihilation flux reported by Knödlseder et al. (2005).

The microquasars can have either "misaligned" (highly inclined with respect to the orbital plane) or "normal" (low inclination) jets. The normal microquasars will pour out their positrons into the ISM, where the usual 2-3 photon production processes take place (see Guessoum et al. 2005). In the case of misaligned microquasars (about $15-20 \%$ of the total) we assume, following Butt et al. (2003), that their jets hit the companion $\sim 10 \%$ of the time. Only 1 of the $\sim 2$ annihilation photons will emerge then, the other being absorbed in the companion's atmosphere (see

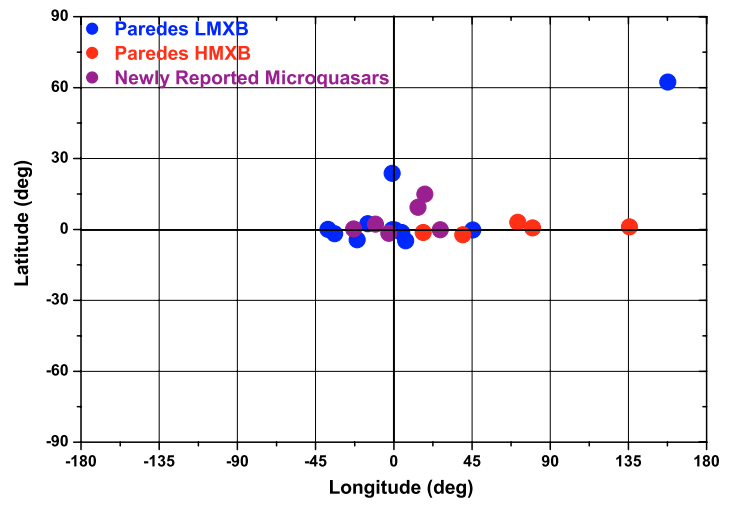

Fig. 1. Microquasars (position and type) in the Galaxy.

Sect. 5). The remaining $90 \%$ of the positrons are thrown into the ISM, where they produce $2-3$ photons each.

The total flux of annihilating positrons coming from the inner Galaxy would be the sum of the flux contributed by the misaligned jets and the normal jets:

$F=F_{\text {mis }}+F_{\text {norm }}$

where

$F_{\text {norm }}=\frac{f_{\mu \mathrm{Q}} N_{\mu \mathrm{Q}}\left(1-f_{\text {mis } \mu \mathrm{Q}}\right) \dot{N}_{\mathrm{e}^{+}}}{4 \pi D^{2}} \times 2 \times f_{\text {line }}$,

and

$$
\begin{aligned}
F_{\mathrm{mis}}= & \frac{f_{\mu \mathrm{Q}} N_{\mu \mathrm{Q}} f_{\mathrm{mis} \mu \mathrm{Q}} \dot{N}_{\mathrm{e}^{+}}}{4 \pi D^{2}} \\
& \times\left[f_{\text {jet }-*}+2 \times\left(1-f_{\text {jet }-*}\right)\right] \times f_{\text {line }},
\end{aligned}
$$

where $N_{\mu \mathrm{Q}}$ is the total number of microquasars believed to exist in the Galaxy $(\sim 100), f_{\mu \mathrm{Q}}$ is the fraction of $\mu \mathrm{Q}$ 's we assume to be in the inner regions of the Galaxy $(\approx 50 \%), f_{\text {mis } \mu \mathrm{Q}}$ is the fraction of $\mu \mathrm{Q}$ 's assumed to have misaligned jets $(\approx 20 \%), f_{\text {jet }-*}$ is the fraction of the time a misaligned jet will hit the companion star's atmosphere $(\approx 10 \%), \dot{N}_{\mathrm{e}^{+}}$is the rate of ejection of positrons from a typical jet $\left(10^{41} \mathrm{~s}^{-1}\right), f_{\text {line }}$ is the fraction of photons emitted with the line energy $(511 \mathrm{keV})$ as opposed to continuum $(0-511 \mathrm{keV})$ energies, and $D$ is the distance to the Galactic centre $(8.5 \mathrm{kpc}) ; f_{\text {line }}$ is obtained from $\frac{1}{4} f_{\mathrm{Ps}}+\left(1-f_{\mathrm{Ps}}\right)$, where $f_{\mathrm{Ps}}$ is the "Positronium fraction", i.e. the fraction of positrons that annihilate via formation of Positronium (the bound $\mathrm{e}^{+}-\mathrm{e}^{-}$system), which has repeatedly been found in galactic annihilation radiation measurements to be $\approx 0.95$ (see references given in Sect. 1).

With those parameter values, we obtain:

$F_{\text {norm }} \approx 4 \times 10^{-4} \mathrm{ph} \mathrm{cm}^{-2} \mathrm{~s}^{-1}$

$F_{\text {mis }} \approx 6 \times 10^{-5} \mathrm{ph} \mathrm{cm}^{-2} \mathrm{~s}^{-1}$.

The total flux $F \approx 5 \times 10^{-4} \mathrm{ph} \mathrm{cm}^{-2} \mathrm{~s}^{-1}$ is within a factor of 2 of the SPI-measured annihilation flux $\left(1.1 \times 10^{-3} \mathrm{ph} \mathrm{cm}^{-2} \mathrm{~s}^{-1}\right)$, a result that is quite encouraging, considering the uncertainties on different parts of the problem (mostly due to our currently limited knowledge of microquasar jet energetics).

As noted in Sect. 1, the spatial distribution of the $511 \mathrm{keV}$ flux detected by SPI-INTEGRAL puts severe constraints on candidate sources of positrons. Although far from complete at present, the available sample of known/confirmed microquasars 
appears encouraging in that respect. Figure 1 displays the position and type (LMXB/HMXB) of the 22 currently known microquasars.

While fully aware that this list represents only about one fifth of the microquasars believed to exist in the Galaxy, we can still attempt to determine a Bulge-to-Disc (B/D) ratio of the annihilation produced by such sources and compare that with the limits obtained from SPI data (Knödlseder et al. 2005), which inferred a rate of positron annihilation of $(1.5 \pm 0.1) \times 10^{43} \mathrm{e}^{+} \mathrm{s}^{-1}$ in the bulge and $(0.3 \pm 0.2) \times 10^{43} \mathrm{e}^{+} \mathrm{s}^{-1}$ in the disc.

Referring to Table 1, which lists the types, positions and distances of these microquasars, we note that roughly $80 \%$ of them are LMXB's, about $15 \%$ of which are in the halo, $35 \%$ are in the disc, and about $50 \%$ are in the bulge, while $20 \%$ of the sources are HMXB's, $\sim 10 \%$ of which may be in the bulge and the rest in the disc. The canonical average rate of positron production has been taken to be about $10^{41} \mathrm{e}^{+} \mathrm{s}^{-1}$ by a typical jet from an LMXB microquasar, while for HMXB microquasars jets are ten times less powerful and thus about three times less productive in positrons (recall that $L_{\mathrm{e}^{+} \mathrm{e}^{-}} \sim L_{\text {jet }} \sim L_{\mathrm{X}}^{0.5}$ ). One must then take into consideration the confinement probability of positrons ejected from these sources; we note that: i) according to Jean et al. (2006), positrons produced in the bulge do not escape, they end up annihilating in the bulge if their energy is below $\sim 10 \mathrm{MeV}$, which is the case for positrons produced by microquasars, if one ignores (as in this basic treatment) internal acceleration processes; ii) the scale height of LMXBs in the disk is $\sim 400 \mathrm{pc}$, while the gas has a scale height of $\sim 100 \mathrm{pc}$, so about $50 \%$ of positrons produced by LMXB's there are ejected toward the disk and annihilate, while the rest $(50 \%)$ of the positrons are released in the halo and either contribute to a diffuse annihilation emission (unseen by spectrometers) or propagate following the galactic magnetic field lines toward the bulge (Prantzos 2006); iii) the escape fraction of positrons produced by HMXB's in the disk is unconstrained, and for simplicity we take it to be the same as that of positrons from disk LMXB's. With these fractions, the net rate of positrons annihilating in the bulge is found to be $\approx 4.1 \times 10^{42} \mathrm{e}^{+} \mathrm{s}^{-1}$ (about one third the SPI bulge rate), while the net rate of positrons annihilating in the disc is found to be $\approx 1.7 \times 10^{42} \mathrm{e}^{+} \mathrm{s}^{-1}$; this would give a B/D ratio of 2.4 , which is somewhat smaller than the lower SPI limit (B/D) SPI $\geq 3$, indicating that positrons produced in the disk are escaping in large(r) fractions than assumed here.

One can also turn these estimates around and set limits on positron production rates from microquasars, knowing that the net rates in the bulge and in the disc cannot exceed those set by SPI as mentioned above. (Note that since we are taking a canonical average value of positron production rate for all LMXB microquasars and assuming the HMXB microquasars to produce only one third as many positrons, for luminosity reasons as explained above, the $\mathrm{B} / \mathrm{D}$ ratio is independent of the jet positron rate and cannot help set constraints on it, at least in our simple model.) Assuming the ratios of LMXB/HMXB microquasars and the escape fractions given in the previous paragraph, we find that the SPI limits would be violated if positrons are produced at steady rates greater than $\sim 3 \times 10^{41} \mathrm{e}^{+} \mathrm{s}^{-1}$. Furthermore, we note that our model easily satisfies the Beacom \& Yüksel (2005) constraint since our positrons have kinetic energies between $2.5 \mathrm{keV}$ and $660 \mathrm{keV}$ (jet speeds range from $0.1 \mathrm{c}$ to $0.9 \mathrm{c}$ ), so their annihilation in flight does not produce continuum emission of photons at higher energies.

Finally, we note that the line profile that can be expected in this scenario is almost exactly the same as the one obtained by SPI and analysed recently (Churazov et al. 2005;
Jean et al. 2006), since the positrons are here poured mostly into the ISM; the small fraction ejected from misaligned microquasars that annihilates on the companion star's surface will produce a line of similar profile, as the physical conditions (temperature and ionization) in the atmospheres of those stars are essentially identical ( $T \sim 8000 \mathrm{~K}, \mathrm{H} / \mathrm{He}$ composition, partially ionized) as those in the ISM phases where the positrons annihilate on large scales.

\section{Annihilation in the atmosphere of the companion star}

In this section we consider in some detail the fate of the jet positrons hitting the atmosphere of the companion star, in the case of misaligned microquasars. Although this happens in a small number of cases $(\sim 15-20 \%$ of all the microquasars, see Sect. 4) and for a small fraction of the XRB's period $(\sim 10 \%)$ there are potentially interesting gamma ray signatures of such an interaction.

Positrons arrive in the companion star with speeds ranging from $0.1 \mathrm{c}$ to $0.9 \mathrm{c}$ (canonical value $v_{\text {jet }} \sim \mathrm{c} / 2$ ), i.e. kinetic energies of $2.5 \mathrm{keV}$ to $660 \mathrm{keV}$. They lose energy in the stellar atmosphere which has a very sharp density profile (see Fig. 1 in Guessoum \& Jean 2002). Using stellar atmosphere models presented in Guessoum \& Jean (2002) we estimate the distance for the energetic positrons to slow down below $60 \mathrm{eV}$ to be $\lambda_{E} \lesssim$ $0.1 \mathrm{~g} \mathrm{~cm}^{-2}$. At this energy, positrons are able to form a positronium by charge exchange with $\mathrm{H}$ atoms. Low energy positrons that do not form a positronium quickly thermalize and annihilate in situ. The maximum atmospheric depth at which positrons annihilate $\left(\lambda_{E}\right)$ is lower than the mean free path of $511 \mathrm{keV}$ photons $\left(\lambda_{511 \mathrm{keV}} \sim 6.6 \mathrm{~g} \mathrm{~cm}^{-2}\right)$ in the atmosphere. Then in these conditions, most $(\gtrsim 0.95 \%)$ of the annihilation photons that are emitted upward do escape the atmosphere of the companion star.

This assumption can be considered valid as long as the jet impact does not strongly deform the stellar atmosphere. It should be noted, however, that the energy deposited by the jets into the star's atmosphere $\left(\sim 10^{36} \mathrm{erg} \mathrm{s}^{-1}\right)$ is so large as to substantially raise the surface temperature (the increase depending on various thermodynamical effects) during the impact periods. While this phenomenon has no bearing on the annihilation of our positrons, it can still lead to some (transitory) observational effects, which may be investigated. Indeed, the depth at which positrons annihilate depends on the thermodynamic conditions of the gas (density, temperature), and using the energy loss rate of positrons in a totally ionized medium (see Eq. (3) of Murphy et al. 2005, and additional references therein), we estimate that the depth at which positrons with initial kinetic energy of $660 \mathrm{keV}$ annihilate is $\sim 0.1 \mathrm{~g} \mathrm{~cm}^{-2}$ for a temperature of $10^{4} \mathrm{~K}$ and $\sim 0.06 \mathrm{~g} \mathrm{~cm}^{-2}$ for a temperature of $10^{6} \mathrm{~K}$. Moreover, at this energy, the range of positron can vary also by a factor of $\sim 3.5$ with the ionization fraction and the abundance (see Fig. 3 of Milne et al. 1999). In any case, the range of $660 \mathrm{keV}$ positron is lower than the mean free path of $511 \mathrm{keV}$ photons $\left(\sim 6.6 \mathrm{~g} \mathrm{~cm}^{-2}\right)$.

Even if a deformation of the surface does occur, the positrons still annihilate at a depth $\sim \lambda_{E}$ and the upward $511 \mathrm{keV}$ photons do escape the atmosphere. However, the impact of the jet produces a cavity which may reduce the escaping solid angle of the upward $511 \mathrm{keV}$ photons. We take this effect into account in an approximate, geometric way.

The jet pressure on the stellar atmosphere is $P_{\text {jet }}=$ $L_{\text {jet }} /\left(v_{\text {jet }} S\right)$, where $L_{\text {jet }}$ is the power of the jet, $v_{\text {jet }}$ its velocity, and $S$ is the impact surface on the secondary. As a first approximation, we consider that the jet stops in the secondary star's 
atmosphere when the jet pressure is equal to the atmospheric gas pressure. At the impact, the jet compresses the atmosphere, producing a cavity with a depth that depends on the pressure profile of the secondary's atmosphere, the jet characteristics (power, velocity and opening angle) and the binary separation. For typical values of jet power $L_{\text {jet }} \sim 10^{36} \mathrm{erg} \mathrm{s}^{-1}$, velocity $v_{\text {jet }} \sim \mathrm{c} / 2$ and opening angle $\theta_{\text {jet }} \sim 1^{\circ}$, we obtain $P_{\text {jet }} \sim 10^{5} \mathrm{erg} \mathrm{cm}^{-3}$, for a binary star separation of $a=2 R_{\odot}$ and a secondary mass of $0.5 M_{\odot}$. Using the density and the temperature profile models of a $0.5 M_{\odot}$ secondary atmosphere (Guessoum \& Jean 2002), we derived the atmospheric gas pressure as a function of the depth and estimated cavity depths (below the photosphere) of $\sim 10^{7} \mathrm{~cm}$.

The fraction of upward $511 \mathrm{keV}$ photons that escape the cavity can be estimated by calculating the opening angle of the cavity (solid angle viewed from the center of the bottom of the cavity to the edge of the top of the cavity). Using the same jet-binary parameters (the radius of a $0.5 M_{\odot}$ is $\sim 0.7 R_{\odot}$ ) and a jet impinging the surface perpendicularly, the radius of the jet impact on the secondary atmosphere is $7.9 \times 10^{8} \mathrm{~cm}$. With a depth of $10^{7} \mathrm{~cm}$, the opening angle is then $\sim 178^{\circ}$, yielding a solid angle of $6.2 \mathrm{sr}$, so the fraction of upward photons that escape the cavity is $98 \%$. Considering the most pessimistic cases (small binary separation and jet impinging perpendicularly on the surface, i.e. high jet pressure), the fraction of upward $511 \mathrm{keV}$ photons escaping the cavity is always larger than $70 \%$ (e.g. with a separation of $a=1.4_{\odot}$, the cavity depth is $\sim 10^{8} \mathrm{~cm}$ and the escaping fraction is $\sim 77 \%$ ). Therefore, we neglect this jet-pressure effect on the secondary atmosphere in the modeling of the $511 \mathrm{keV}$ flux presented in the next paragraphs.

We also checked that the magnetic field of the secondary does not affect the jet: we compared the jet pressure with the magnetic pressure $P_{B}=B^{2} /(4 \pi)$; taking a most extreme value for the stellar magnetic field $B \sim 10^{3} \mathrm{G}$ we obtain $P_{B} \sim$ $8 \times 10^{4} \mathrm{erg} \mathrm{cm}^{-3}$ which is lower than $P_{\text {jet }}$.

The upward $511 \mathrm{keV}$ photons that escape the atmosphere may enter the incoming jet. They traverse the jet if their mean free path is larger than the jet dimension. The minimum mean free path of $511 \mathrm{keV}$ photons in the jet material is $\lambda>\left(n_{\mathrm{e}} \sigma_{\mathrm{T}}\right)^{-1}$, with $n_{\mathrm{e}}$ the electron plus positron density in the jet and $\sigma_{\mathrm{T}}$ the Thompson cross-section. At the secondary surface, $n_{\mathrm{e}}$ can be approximated by $n_{\mathrm{e}} \sim 2 \dot{N}_{\mathrm{e}^{+}} /\left(v_{\text {jet }} S\right)$. Assuming a close binary system (e.g. $a=2 R_{\odot}$ and $R_{2}=0.7 R_{\odot}$ ) to estimate the smallest impact area $S$ and therefore the largest density $n_{\mathrm{e}}$, we obtain $\lambda>$ $6 R_{\odot}$ (assuming $v_{\text {jet }} \sim \mathrm{c} / 2$ and $\theta_{\text {jet }} \sim 1^{\circ}$ ). This lower limit is already larger than the size of the XRB separation. Consequently, the jet itself can be considered as quasi-transparent to those gamma photons and the general conclusion is that positrons annihilate near the surface, and about half the resulting $511 \mathrm{keV}$ photons will be emitted upwards, towards the surface and the incoming jet.

The binary system rotates, and the secondary star periodically crosses the jet, which slowly precesses. Consequently, the $511 \mathrm{keV}$ photon radiation flux received by an observer in a given direction is a function of time, and its intensity and timevariability characteristics depend on the parameters of the XRB (period, inclination, distance, separation and radius of the secondary star) and of the jet (inclination with respect to the binary plane, opening angle and precession period). The flux at $511 \mathrm{keV}$ can be calculated using the following expression:

$F_{511 \mathrm{keV}}(t)=2 \times\left(1-\frac{3}{4} f_{\mathrm{Ps}}\right) \times \dot{N}_{\mathrm{e}^{+}} \times \frac{I(t)}{4 \pi d^{2}}$

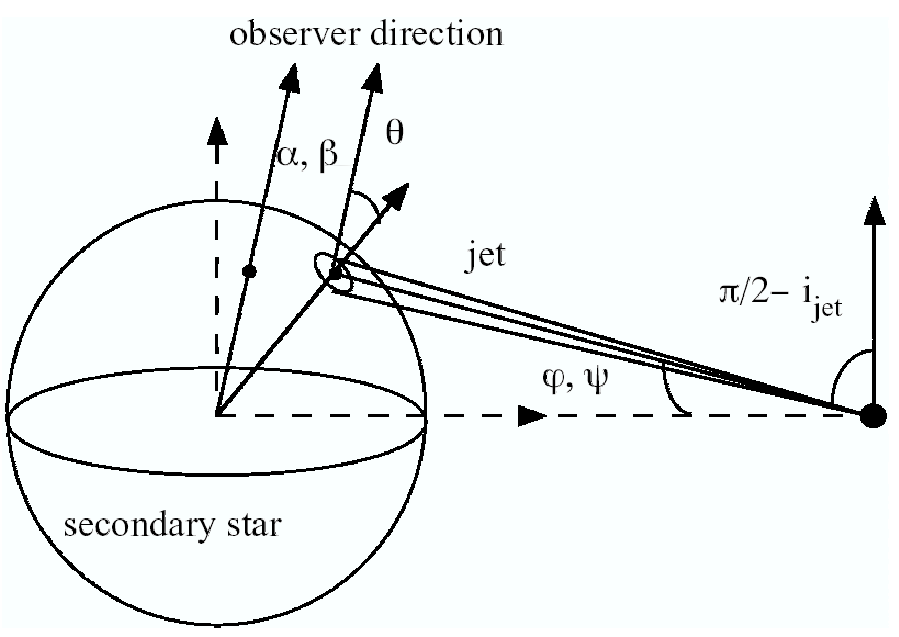

Fig. 2. Schematic view of the irradiation of the secondary star by a jet. The binary system frame is represented by the dashed axes.

where $f_{\mathrm{Ps}}$ is the positronium fraction, $\dot{N}_{\mathrm{e}^{+}}$is the rate of ejection of positrons from the jet, and $d$ is the distance of the binary system to the observer. The function $I(t)$ is the fraction of $511 \mathrm{keV}$ photons coming from the atmosphere in the direction of the observer. This function takes into account the fraction of the jet's solid angle (which contains the positrons) that (i) intersects the secondary star surface and (ii) is visible by the observer. Its expression is given by:

$I(t)=\frac{1}{\Delta \Omega_{\text {jet }}} \int_{(\varphi, \psi) \in D(t)} \mathrm{e}^{-\frac{\lambda_{E}(\varphi, \psi)}{\lambda_{511} \operatorname{keV} \cos \theta(\varphi, \psi)}} \mathrm{d} \Omega(\varphi, \psi)$

where $\lambda_{E}$ is the atmospheric depth (in $\mathrm{g} \mathrm{cm}^{-2}$ ) at which annihilation occurs, and $\lambda_{511 \mathrm{keV}}$ is the mean free path of $511 \mathrm{keV}$ photons in the stellar atmosphere. $\Delta \Omega_{\text {jet }}$ is the solid angle of the jet; $\varphi$ and $\psi$ give the direction of the jet (see Fig. 2); and $D(t)$ is the integration domain $(\varphi, \psi \in D(t))$, defined as the intersection of the irradiated area and the visible area in the direction of the observer (the set of directions $\varphi$ and $\psi$ for which $\theta<90^{\circ}$ providing the observer direction $\alpha$ and $\beta$ ). The irradiation area (or hotspot) is delimited by the intersection of the companion surface with the cone of angle $\theta_{\text {jet }} / 2$ around the jet direction $\varphi_{\text {jet }}$ and $\psi_{\text {jet }}$ (see Fig. 2). In Eq. (9), $\theta$ is also a function of $\alpha, \beta$, the XRB separation $(a)$ and the secondary radius $\left(R_{2}\right)$. Since $I(t)$ is expressed in the XRB frame that is rotating with respect to the observer, $\alpha$, $\varphi_{\text {jet }}$ and $\psi_{\text {jet }}$ vary with time, while $\beta$, the angle between the binary system plane and the observer direction, is equal to $90^{\circ}-i$ ( $i$ is the "official" inclination of the XRB).

To illustrate the temporal variations of the annihilation flux, we calculated the $511 \mathrm{keV}$ light curve for the case of a "prototypical misaligned microquasar", one that has similar parameters (separation, inclination, primary and secondary masses...) as GRO J1655-40, which is one of two XRB's for which independent information is available on the jet orientation (Narayan $\&$ McClintock 2005). However, we arbitrarily assumed a jet inclination of $i_{\text {jet }}=9^{\circ}$ with respect to the binary plane. With such an inclination, the jets hit the companion star at about half of its upper hemisphere. We also assumed an opening angle $\theta_{\text {jet }}=1^{\circ}$ and a precession period of 300 days which is the order of magnitude of the precession period of such an inclined jet (Kaufman Bernadó et al. 2002).

Could there be some observational effects on the radio emission from the interaction of the jet with the surface of the star? In this regard, we first note that pairs in the jets will emit synchrotron radiation for most (say 90\%) of their transit (ejection) 


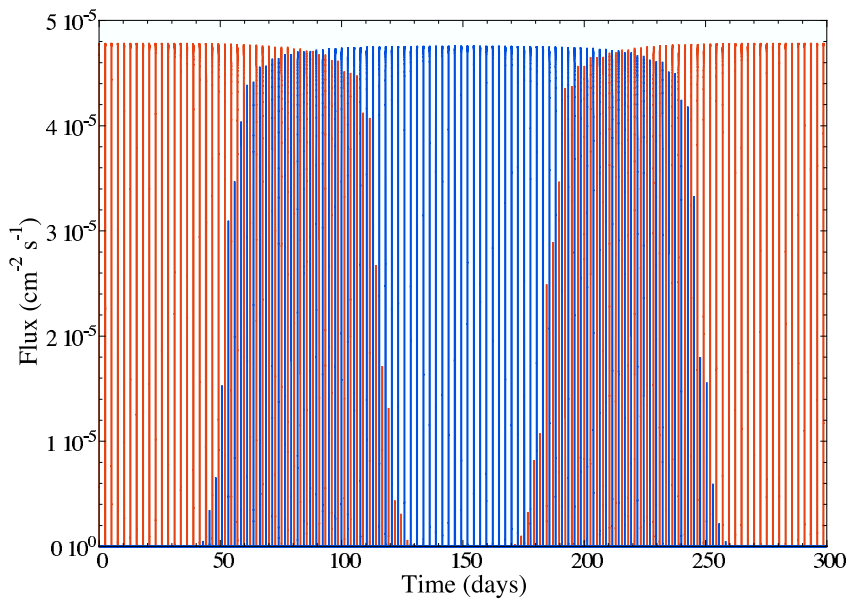

Fig. 3. Instantaneous $511 \mathrm{keV}$ photon flux induced by the jet (red curve) and the counterjet (blue curve) impacts on the secondary of a virtual XRB (see text). The jet inclination with respect to the binary plane is taken to be $i_{\text {jet }}=9^{\circ}$, its opening angle is $\theta_{\text {jet }}=1^{\circ}$; the precession period is 300 days (see text). The positron rate and energy in each jet is $\dot{N}_{\mathrm{e}^{+}}=$ $10^{41} \mathrm{~s}^{-1}$ and $E=660 \mathrm{keV}\left(v_{\text {jet }}=0.9 \mathrm{c}\right)$, respectively.

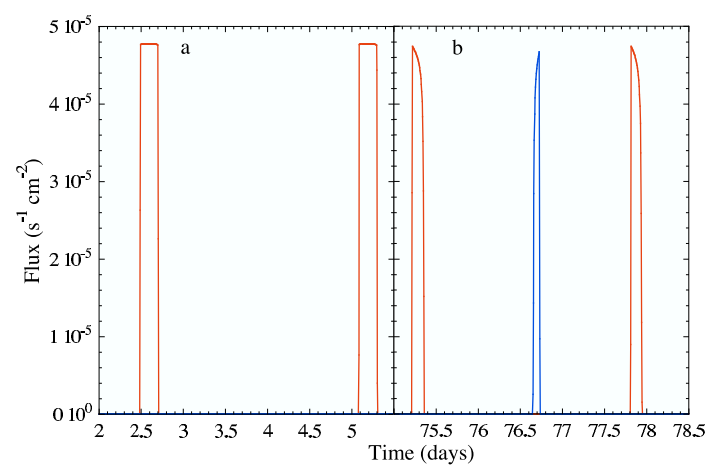

Fig. 4. Details of the instantaneous $511 \mathrm{keV}$ photon flux induced by the jet (red curve) and the counterjet (blue curve) impacts on the secondary of a virtual XRB (see caption of Fig. 3). a) The jet direction is parallel to the observer direction and only the hotspot induced by the first jet is visible; b) the jet direction is perpendicular to the observer direction and both hotspots induced by the jet and the counterjet are visible. The geometrical configurations of the jets and the binary system in these two cases are shown in Fig. 5.

time, while the rest of the time they spend depositing their energies in the companion's atmosphere; this energy is lost first and foremost in the form of heat and then, at the end, as gamma rays, which get downgraded as X-rays and again as heat. It is thus not expected that much radio emission will come out of the jet's collision with the companion star's atmosphere, but this issue could be investigated further in the future.

As for GRO J1655-40, this "prototypical” microquasar was assumed to be at a distance of $3.2 \mathrm{kpc}$ from Earth, to have a period of 2.62 days and to be composed of a compact object with a mass of $6.8 M_{\odot}$ separated by $16.6 R_{\odot}$ from a companion star with a radius of $5 R_{\odot}$. The inclination of the binary system is $i=70^{\circ}$. Figure 3 shows the instantaneous $511 \mathrm{keV}$ flux emitted by such a microquasar as a function of time. Details of this light curve are presented in Fig. 4 for two different dates: (a) when the direction of the jets lies approximately along the line of sight, and in (b) when the direction of the jets is approximately perpendicular to the line of sight. The geometrical configurations of the jets and the binary system in these two cases are shown in Fig. 5.

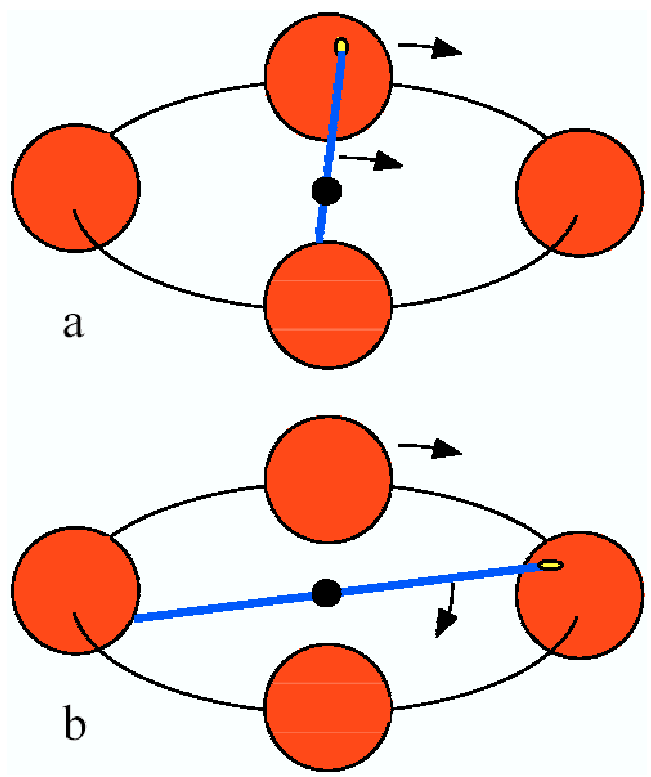

Fig. 5. Schematic views of the jets (blue) arising from the compact object (black), the secondary star (red) presented at four positions in its orbit, and the annihilation hotspot (yellow) emitted from the secondary star atmosphere, presented at the dates $\sim 2$ days a) and $\sim 75$ days b). The directions of motion of the secondary star and jets axis are represented by arrows. The two configurations correspond approximately to the cases a and b of Fig. 4.

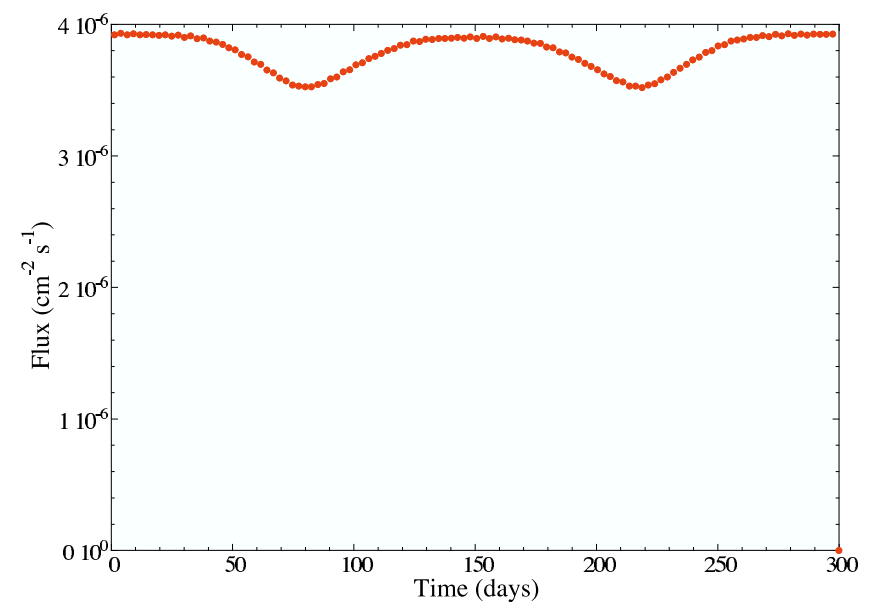

Fig. 6. Average $511 \mathrm{keV}$ photon flux per XRB period, induced by the jet impacts on the secondary of an XRB. The parameters of the jets are identical to those of Figure.

Figure 6 shows the time series of the $511 \mathrm{keV}$ flux averaged per period of the XRB. The flux is minimum when the jet direction is perpendicular to the observer direction, as shown in Figure $5 \mathrm{~b}$. In this configuration, the inclination $\theta$ of the direction of the photons emitted by the fraction of the hotspot that is visible can be close to $90^{\circ}$ during the crossing of the secondary. Consequently, a large fraction of these photons are absorbed in the atmosphere of the secondary (see Eq. (9) and Fig. 4b). Note that the average flux is $\sim 10$ times lower than the maximum of the instantaneous flux since the companion star crosses the jets during a fraction $\sim 10 \%$ of the period.

The spectral shape of the annihilation radiation emitted by this virtual microquasar was calculated accordingly to the model of Guessoum et al. (2005), which provides annihilation spectra as a function of the physical conditions of the gas in which 


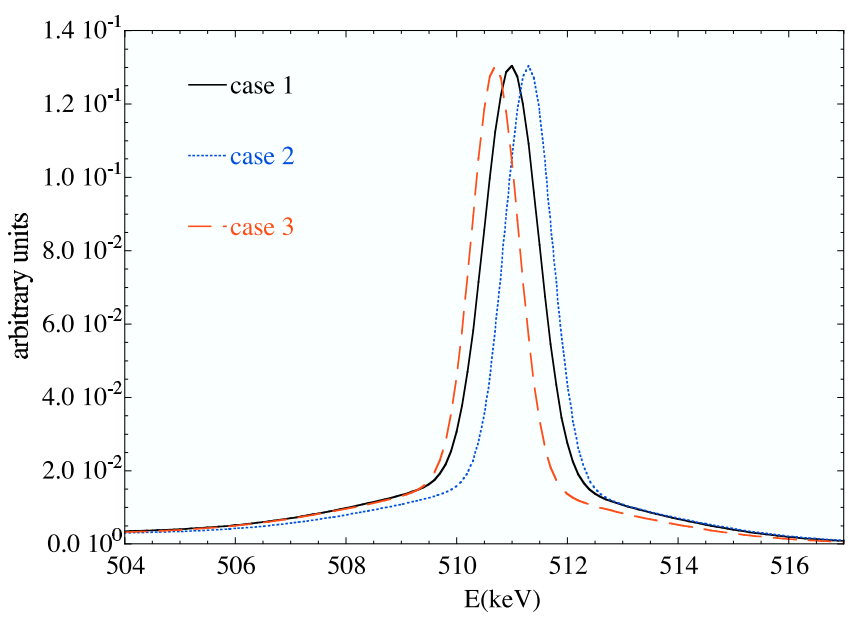

Fig. 7. Spectra of the annihilation radiation emitted by GRO J1655-40. The parameters of the jets are identical to those of Fig. 3. We assumed that the $50 \%$ of positrons annihilates with the ionized component of the atmosphere and $50 \%$ with the neutral component. Case 1: total spectrum integrated over the precession period; case 2: spectrum integrated over one period at the date 100 days; case 3: spectrum integrated over one period at the date 200 days.

the annihilation occurs. We assumed that positrons annihilate in a half-ionized and half-neutral plasma with a temperature of $8000 \mathrm{~K}$. The rotation of the binary system leads to a shift in the centroid of the $511 \mathrm{keV}$ line. This Doppler shift is a function of time and depends on the observer direction. We calculated this spectral shift using the method described in Jean \& Guessoum (2001). Figure 7 shows the spectra: integrated over the precession period (case 1); integrated over one XRB period at $t=100$ days (case 2, see Fig. 3); integrated over one XRB period at $t=200$ days (case 3 ). The line is blue-shifted by $0.3 \mathrm{keV}$ in case 2 because most of the annihilation emission comes from the jet impact on the secondary which is moving toward the observer. In case 3 , most of the annihilation emission comes from the jet impact on the secondary which is receding from the observer, thus producing a line with a red-shift of $0.3 \mathrm{keV}$.

The results presented in this section show that the measurements of the temporal and spectral profiles of the annihilation radiation emitted by microquasars with misaligned jets are useful to probe the characteristics of the jet. The maximum $511 \mathrm{keV}$ instantaneous flux provides the rate of positrons in the jet (e.g. Fig. 3). The duration of the $511 \mathrm{keV}$ emission in one XRB period allows one to estimate the opening angle of the jet (e.g. Fig. 4), while the long-term variation of the average flux allows for a determination of the precession period of the jet (e.g. Fig. 6). The spectral shape of the $511 \mathrm{keV}$ line, do, in principle, allow one to infer the physical conditions of the plasma in which positrons annihilate, as Jean et al. (2006) demonstrated for the SPI data of positron annihilation in the ISM, although one must realize that in more complex situations a host of conditions and effects can render the extraction of physical information very complicated if not impossible.

Finally, in Table 2 we present our predicted maximum fluxes for the confirmed misaligned microquasars as well as for potential candidates, i.e. microquasars that may be at least occasionally misaligned and are not too far away for detection by future instruments. We must note that the values given in this table are for "average jets"; "strong-flare jets" could be 10 times more powerful, thus producing 10 times more positrons and thus resulting in an annihilation flux 10 times higher (than what is
Table 2. Predicted maximum $511 \mathrm{keV}$ emission fluxes for confirmed or potentially misaligned nearby microquasars. Fluxes were calculated for "Average Jet", that is using a positron rate of $10^{41} \mathrm{e}^{+} \mathrm{s}^{-1}$.

\begin{tabular}{lccc}
\hline \hline \multicolumn{1}{c}{ Source } & Jet & $\begin{array}{c}\text { Distance } \\
(\mathrm{kpc})\end{array}$ & $\begin{array}{c}\text { Flux for “Average Jet" } \\
\left(10^{-4} \mathrm{~cm}^{2} \mathrm{~s}^{-1}\right)\end{array}$ \\
\hline GRO J1655-40 & Misaligned & 3.2 & 0.49 \\
V4641 Sgr & Misaligned & 9.6 & 0.05 \\
XTE J1550-564 & Misaligned? & 5.3 & 0.18 \\
XTE J1118+480 & & 1.8 & 1.53 \\
LS I +61 303 & 2 & 1.24 \\
Cyg X-1 & 2.5 & 0.79 \\
Sco X-1 & 2.8 & 0.63 \\
LS 5039 & 2.9 & 0.59 \\
SS 433 & 4.8 & 0.22 \\
GRS 1758-258 & 8.5 & 0.07 \\
Cyg X-3 & 9 & 0.06 \\
Cir X-12 & & 10 & 0.05 \\
GRS 1915+105 & & 12.5 & 0.03 \\
GX 339-4 & $\geq 8$ & $\leq 0.08$ \\
XTE J1748-288 & $\geq 8$ & $\leq 0.08$ \\
1E 1740.7-2942 & 8.5 & 0.07 \\
IGR J17091-3624 & $8.5 ?$ & 0.07 \\
IGR J17303-0601 & $8.5 ?$ & 0.07 \\
IGR J17464-3213 & $8.5 ?$ & 0.07 \\
IGR J18406-0539 & $8.5 ?$ & 0.07 \\
\hline
\end{tabular}

given in the table); on the other hand, "weak episode jets" would give positron rates and annihilation fluxes about 10 times lower. Indeed, the annihilation flux is directly proportional to the jet power.

\section{Summary}

The origin of the Galactic positron annihilation radiation is a major issue in high energy astrophysics at present. In this work we assess the potential of Galactic microquasars (XRBs that exhibit jets in an intermittent way) as sources of positrons.

Among the rapidly growing body of data on microquasars, we present in Sect. 2 the main features, those of relevance to our study. The correlation between the power of the jet and the X-ray luminosity of the compact object is the most important of these features. We stress, in particular, that the ratio between the two, often assumed to be small, is highly uncertain; moreover, the content of the jets, leptonic or baryonic (i.e. electron-positron pairs vs. protons and pions), is unknown at present. Some of the implications of a baryonic content have been explored elsewhere (Butt et al. 2003; Romero \& Orelana 2005); we explore here the consequences of a leptonic content.

In Sect. 3 we evaluate the rate of positron ejection by the microquasar jets, based on various models proposed in the literature, but also on simple energetic arguments relating the total power of the positrons in the jets to the estimated total X-ray luminosity of the "low luminosity - hard spectrum" galactic LMXRBs. We find that a value of $10^{41} \mathrm{e}^{+} \mathrm{s}^{-1}$ could be considered as a "canonical average", albeit with large (and difficult to evaluate) uncertainties.

In Sect. 4 we estimate the total positron production rate by the collective emission of Galactic microquasars and find it smaller (about one third) than what is inferred from SPI/INTEGRAL observations of $511 \mathrm{keV}$ radiation. The spatial morphology of the corresponding flux received on Earth depends on the assumed large scale distribution of Galactic microquasars, which is very poorly known at present; we find that the 
distribution of the currently available (incomplete) sample appears encouraging, in that respect. (We should also note that a large enough $\mathrm{B} / \mathrm{D}$ ratio would be obtained with any source distributions that congregate in the bulge and/or suppress the annihilation of their positrons in the disk.) Finally, we constrain the rate of production of positrons by microquasars on the basis of the SPI flux results: we find that the SPI limits would be violated if positrons are produced at steady rates greater than $\sim 3 \times 10^{41} \mathrm{e}^{+} \mathrm{s}^{-1}$.

Finally, in Sect. 5 we present an investigation of the physics of "misaligned" microquasars, where the jet impinges on the companion star. We find that about half of the annihilation photons should, in general, escape the stellar atmosphere. We compute fluxes for a list of sources (see Tab. 2), a few of them known to be misaligned, and some of the others may turn out to be so as well. The expected annihilation fluxes are found to be low, however, and difficult to measure with the spectrometer SPI due to the distance of most of the "misaligned" microquasars. Future instruments such as a gamma-ray lens (von Ballmoos et al. 2004) or a Compton telescope (Boggs \& Jean 2001) would have the sensitivity required to measure these fluxes. Due to the rotation of the binary system, the secondary star periodically crosses the jet, leading to a "periodic" and a Doppler-shifted annihilation emission. Due to the precession of the jet, we expect to observe a long-term modulation of the emission. A detection of a point source at $511 \mathrm{keV}$ from a "misaligned" microquasar, with such temporal and spectral signatures would confirm the presence of positrons in the jet. Moreover, such a measurement would allow for a determination of the rate of positrons channeled by the jet and the characteristics (opening angle, precession period) of the jet.

In summary, we have shown in this study that microquasar jets may constitute important sources of annihilation radiation, both diffuse (for most of them) or point sources (in the case of misaligned ones).

Acknowledgements. We wish to acknowledge helpful discussions with Didier Barret and Alexandre Marcowith. We are grateful to Mamta Pandey for bringing to our notice the four new microquasars that she and her collaborators have recently reported. And we thank the anonymous referee for several useful suggestions and requests for clarifications, which has led to a definite improvement of the paper.

\section{References}

Ascasibar, Y., Jean, P., Boehm, C., \& Knödlseder, J. 2006, MNRAS, 368, 1695 Beloborodov, A. M. 1999, MNRAS, 305, 181

Beacom, J. F., \& Yüksel, H. 2006, Phys. Rev. Lett., 97, 071102

Boehm, C., Hooper, D., Silk, J., Casse, M., \& Paul, J. 2004, Phys. Rev. Lett. 92, 1301

Boggs, S. E., \& Jean P. 2001, A\&A, 366, 1126

Bosch-Ramon, V., \& Paredes, J. M. 2004a, A\&A, 417, 1075

Bosch-Ramon, V., \& Paredes, J. M. 2004b, A\&A, 425, 1069

Bosch-Ramon, V., Romero, G. E., Paredes, J. M. 2005, A\&A, 429, 267

Butt, Y. M., Maccarone T. J., Prantzos N. 2003, ApJ, 587, 748

Cassé, M., Cordier B., Paul J., \& Schanne S. 2004, ApJ, 602, 17

Celotti, A., \& Blandford, R. D. 2001, in Proc. of the ESO Workshop in Honour of Riccardo Giacconi, ESO Astrophysics Symposia, ed. L. Kaper, E. P. J. van den Heuvel, \& P. A. Woudt (Springer-Verlag), 206

Chaty, S. 2005, in Proc. of Rencontres de Moriond, Very High Energy Phenomena in the Universe, La Thuile, Italy (March 12-19, 2005), [arXiv:astro-ph/0506008]

Cheng, L. X., Leventhal, M., Smith, D. M., et al. 1997, ApJ, 481, L43
Churazov, E., Sunyaev R., Sazonov S., Revnivtsev M., \& Varshalovich D. 2005, MNRAS, 357, 1377

Clayton, D. D. 1973, Nature Phys. Sci., 244, 137

Clayton, D. D., \& Hoyle F. 1974, ApJ, 187, 101

Dearborn, D. S. P., \& Blake J. B. 1985, ApJ, 288, 21

Dermer, C. D., \& Böttcher, M. 2006, ApJ, 643, 1081

Diehl, R., Prantzos N., \& von Ballmoos P. 2005, Nucl. Phys. A, in press [arXiv: astro-ph/0502324]

Falcke, H., Körding E., \& Markoff S. 2004, A\&A, 414, 895

Fender, R. P., Belloni T. M., \& Gallo E. 2004, MNRAS, 355, 1105

Fender, R. P., Belloni T. M., \& Gallo E. 2005a, Ap\&SS, 300, 1

Fender, R. P., Maccarone T. J., \& van Kesteren Z. 2005b, MNRAS, 360, 1085

Grimm, H. J., Gilfanov M., Sunyaev R. 2002, A\&A, 391, 923

Guessoum, N., \& Jean P. 2002, A\&A, 396, 157

Guessoum, N., Jean P., Knodlseder J., et al. 2004, Proc. 5th INTEGRAL workshop (Munich), ESA SP-552, 57

Guessoum, N., Jean P., \& Gillard W. 2005, A\&A, 436, 171

Heinz, S., \& Sunyaev R. 2002, A\&A, 390., 751

Jean, P., \& Guessoum N. 2001, A\&A, 378, 509

Jean, P., Knodlseder J., Gillard W., et al. 2006, A\&A, 445, 579

Johnson, W. N. III, Harnden F. R. Jr., \& Haymes R. C. 1972, ApJ, 172L, 1

Kaiser, C. R., \& Hannikainen D. C. 2002, MNRAS, 330, 225

Kaufman Bernadó, M. M., Romero G. E., \& Mirabel I. F. 2002, A\&A, 385, L10

Klein-Wolt, M., Homan J., \& van der Klis M. 2004, Nuclear Physics B (Proc. Suppl.), 132, 381

Knödlseder, Jean, P., Lonjou, V., et al. 2005, A\&A, 441, 513

Lingenfelter, R. E., \& Hueter G. J., 1984, in High-Energy Transients in Astrophysics, ed. S. E. Woosley, AIP Conf. Proc., 558

Liu, Q. Z., van Paradijs J., \& van den Heuvel E. P. J. 2000, A\&A S, 147, 25

Liu, Q. Z., van Paradijs J., \& van den Heuvel E. P. J. 2001, A\&A, 368, 1021

Marshall, H. L., Canizares C. R., \& Schulz N. S. 2002, ApJ, 564, 941

Meier, D. 1996, ApJ 459, 185

Milne, P. A., Kurfess J. D., Kinzer R. L., et al. 2000, AIP Conf. Proc., 510, 21

Milne, P. A., Kurfess, J. D., Kinzer, R. L., \& Leising M. D., Dixon D. D. 2001, AIP Conf. Proc., 587, 11

Mirabel, I. F. 2004, Proc. 5th INTEGRAL workshop (Munich), ESA SP-552, 175

Mirabel, I. F., \& Rodriguez L. F. 1994, Nature, 371, 46

Mirabel, I. F., Rodriguez L. F., Cordier B., Paul J., \& Lebrun, F. 1992, Nature, 358,215

Mirabel I. F., Rodriguez L. F., \& Chaty S. 1996, ApJ, 472, L111

Misra, R., \& Melia M. 1993, ApJ, 419L, 25

Murphy R. J., Share, G. H., Skibo, J. G., \& Kozlovsky, B. 2005, ApJS, 161, 495

Milne, P. A., The, L.-S., \& Leising, M. D. 1999, ApJS, 124, 503

Narayan, R., \& McClintock J. E. 2005, ApJ, 623, 1017

Norgaard, H. 1980, ApJ, 236, 895

Pandey, M., Manchanda, R. K., Rao, A. P., Durouchoux, P., \& Ishwara-Chandra 2006a, A\&A, 446, 471

Pandey, M., Rao, A. P., Pooley, G. G., et al. 2006b, A\&A, 447, 525

Paredes, J. M. 2005, Chin. J. Astron. Astrophys., 5, 121

Prantzos, N. 2004, Proc. 5th INTEGRAL workshop (Munich), ESA SP-552, 15

Prantzos, N. 2006, A\&A, 449, 869

Prantzos, N., \& Cassé, M. 1986, ApJ 307, 324

Purcell, W. R., et al. 1994, in Proc. Second Compton Symp., eds. C. Fichtel, N. Gehrels, \& J. Norris (New York: AIP), 403

Purcell, W. R., Cheng, L.-X., Dixon, D. D., et al. 1997, ApJ, 491, 725

Ramaty, R., Stecker F. W., \& Misra, D. 1970, J. Geophys. Res., 75, 1141

Ramaty, R., \& Lingenfelter R. E. 1979, Nature, 278, 127

Ribò, M. 2005, in Future Directions in High Resolution Astronomy: A Celebration of the 10th Anniversary of the VLBA, ed. J. D. Romney, \& M. J. Reid, ASP Conf. Ser., 340, 269

Romero, G. E., \& Orellana, M. 2005, A\&A, 436, 237

Rudaz, S., \& Stecker, F. W. 1988, ApJ, 325, 16

Scheck, L., Aloy M. A., Marí, J. M., Gómez J. L., \& Mller E. 2002, MNRAS, 331,615

Sturrock, P. A. 1971, ApJ, 164, 529

Titarchuk, L., \& Chardonnet, P. 2006, ApJ, 641, 293

Tsarevsky, G. S. 2004, The Astronomer's Telegram, \# 239

von Ballmoos, P., Halloin, H., Skinner, G. K., et al. 2004, SPIE, 5168, 482

Wang, W., Pun C. S. J., \& Cheng K. S. 2006, A\&A, 446, 943

Yamasaki, T., Takahara F., \& Kusunose, M. 1999, ApJ, 523L, 21

Zdziarski, A. A., Coppi P. S., \& Lamb D. Q. 1990, ApJ, 357, 149 